TITLE:

\title{
Surface velocity divergence model of air/water interfacial gas transfer in open-channel flows
}

$\operatorname{AUTHOR}(\mathrm{S})$ :

Sanjou, M.; Nezu, l.; Okamoto, T.

\section{CITATION:}

Sanjou, M....[et al]. Surface velocity divergence model of air/water interfacial gas transfer in open-channel flows. Physics of Fluids 2017, 29: 045107.

\section{ISSUE DATE:}

2017-04-01

URL:

http://hdl.handle.net/2433/252304

RIGHT:

(C) 2017 Author(s).; 許諾条件に基づいて掲載しています。 


\section{Surface velocity divergence model of air/ water interfacial gas transfer in open- channel flows}

Cite as: Phys. Fluids 29, 045107 (2017); https://doi.org/10.1063/1.4981228

Submitted: 04 December 2016 . Accepted: 27 March 2017 . Published Online: 28 April 2017

M. Sanjou (D, I. Nezu, and T. Okamoto
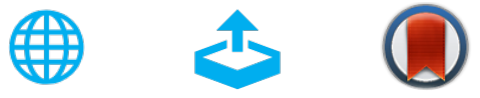

View Online

Export Citation

\section{ARTICLES YOU MAY BE INTERESTED IN}

Vorticity budgets in premixed combusting turbulent flows at different Lewis numbers Physics of Fluids 29, 045106 (2017); https://doi.org/10.1063/1.4981219

Implicit large eddy simulation of acoustic loading in supersonic turbulent boundary layers Physics of Fluids 29, 046101 (2017); https://doi.org/10.1063/1.4979965

Sensitivity of hydrothermal wave instability of Marangoni convection to the interfacial heat transfer in long liquid bridges of high Prandtl number fluids

Physics of Fluids 29, 044105 (2017); https://doi.org/10.1063/1.4979721

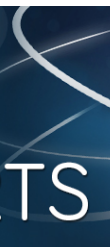

Explore the latest discoveries in your field of research 


\title{
Surface velocity divergence model of air/water interfacial gas transfer in open-channel flows
}

\author{
M. Sanjou, I. Nezu, and T. Okamoto \\ Department of Civil and Earth Resources Engineering, Kyoto University, Kyoto 6158540, Japan
}

(Received 4 December 2016; accepted 27 March 2017; published online 28 April 2017)

\begin{abstract}
Air/water interfacial gas transfer through a free surface plays a significant role in preserving and restoring water quality in creeks and rivers. However, direct measurements of the gas transfer velocity and reaeration coefficient are still difficult, and therefore a reliable prediction model needs to be developed. Varying systematically the bulk-mean velocity and water depth, laboratory flume experiments were conducted and we measured surface velocities and dissolved oxygen (DO) concentrations in openchannel flows to reveal the relationship between DO transfer velocity and surface divergence (SD). Horizontal particle image velocimetry measurements provide the time-variations of surface velocity divergence. Positive and negative regions of surface velocity divergence are transferred downstream in time, as occurs in boil phenomenon on natural river free-surfaces. The result implies that interfacial gas transfer is related to bottom-situated turbulence motion and vertical mass transfer. The original SD model focuses mainly on small-scale viscous motion, and this model strongly depends on the water depth. Therefore, we modify the SD model theoretically to accommodate the effects of the water depth on gas transfer, introducing a non-dimensional parameter that includes contributions of depth-scale large-vortex motion, such as secondary currents, to surface renewal events related to DO transport. The modified SD model proved effective and reasonable without any dependence on the bulk mean velocity and water depth, and has a larger coefficient of determination than the original SD model. Furthermore, modeling of friction velocity with the Reynolds number improves the practicality of a new formula that is expected to be used in studies of natural rivers. Published by AIP Publishing. [http://dx.doi.org/10.1063/1.4981228]
\end{abstract}

\section{INTRODUCTION}

Various aquatic ecosystems establish themselves in natural rivers and basins. Of particular importance is gas transfer across the free-surface in open-channel flows. The distribution of dissolved oxygen (DO) gas in natural rivers is a significant indicator of water quality. Gas transfer across the air-water interface has been studied for decades, and numerous conceptual models have been proposed. Many of these models, including very recent ones, are based on the surface renewal (SR) model of Danckwerts. ${ }^{1}$ The SR model assumes that a fluid parcel in the free surface layer is periodically replaced by local turbulence. A large SR rate ensures that a fresh fluid parcel always exists at the free surface; hence, oxygen from the atmosphere is thoroughly dissolved. Mathematical modeling of the renewal rate is an important research topic. McCready et $a l .^{2}$ proved that the divergence of the freesurface velocity is closely related to gas transfer through the air-water interface. With innovative developments in particle image velocimetry (PIV) measurements, we can obtain the planar distribution of the two velocity components, thereby enabling the calculation of the surface velocity divergence. Therefore, the surface divergence (SD) is of great interest in gas transfer studies. The significance of the peak spectrum of the SD value was highlighted by Tamburrino and Gulliver. ${ }^{3}$ Banerjee et al.$^{4}$ conducted direct numerical simulations of the SD model and reported that it applies not only to open-channel flows but also to free-surface currents induced by wind shear.

To validate the SD model in open-channel and windshear flows, Turney and Banerjee ${ }^{5}$ measured the SD with a stereoscopic PIV system. The SD model was very close to the open-channel results, but it could not capture the wind-shear flow. By recognizing that the time scale of the SD produced by wind-shear is sufficiently small to promote gas transfer, Turney and Banerjee ${ }^{5}$ predicted the gas transfer velocity with a reasonable accuracy in wind-shear flows by combining the SD and SR models. They concluded that the SD model can accurately predict open-channel flows but is limited to windless conditions.

Although the velocity shear is comparatively small near the free surfaces of open-channel flows such as natural rivers, the turbulent motions generated in the bottom layer are nonnegligible. Figure 1 shows a sketch of air/water interfacial gas transfer and the related turbulence structure in open-channel flows. Open-channel turbulence exemplifies a fully developed turbulent boundary layer and exhibits various coherent structures such as hairpin vortices (Robinson ${ }^{6}$ ) and bursting phenomena (Nezu and Nakagawa ${ }^{7}$ ). When conveyed toward the free-surface, these structures have striking impacts on it, similar to the effects of boil vortices on gas transfer. The applicability of gas transfer models to open-channel flows has been examined in several studies. Thackston and $\mathrm{Krenkel}^{8}$ predicted the reaeration coefficient from the bed friction velocity and 


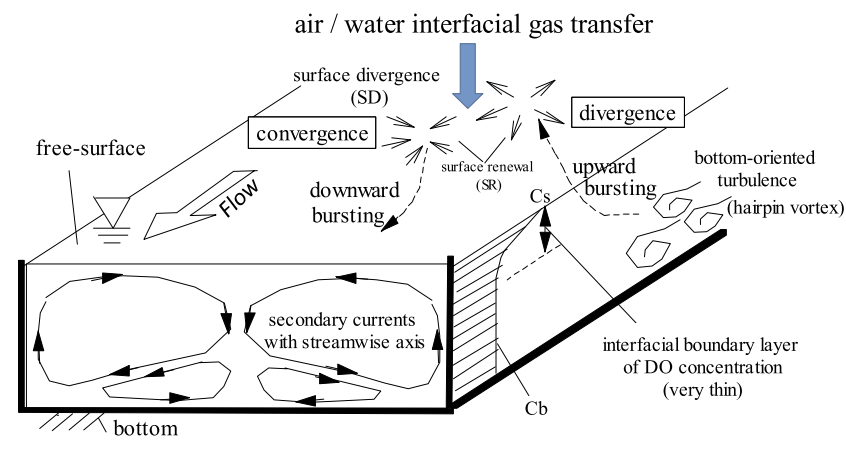

FIG. 1. Phenomenological model of air/water interfacial gas transfer and surface velocity divergence in open-channel flows.

water depth. Gulliver and Halverson ${ }^{9}$ measured the gas transfer velocity in a laboratory flume. They induced water currents using a moving belt situated at the bottom of the flume and noted the significant role of coherent events in turbulence such as ejections and sweeps. Komori et al. ${ }^{10}$ studied the relationship between bottom-generated bursting and SR vortices and revealed the contributions of coherent turbulence events in gas transfer. Moog and Jirka ${ }^{11}$ suggested that the gas transfer phenomenon observed in open-channel flows is consistent with a small-eddy model, in which the gas transfer velocity is normalized by a small-scale static turbulence parameter such as dissipation rate.

Theoretical models cannot generally reproduce gas transfer velocities in river flows. Hence, most river engineers depend on empirical formulae to compute such velocities from parameters such as mean velocity and water depth. However, these practical methods disregard the effects of bursting motion and secondary currents as indicated in Fig. 1. In particular, free-surface turbulence is influenced not only by the mean velocity and water depth but also by the bed configuration and aspect ratio of the water channel. For example, it is well known that submerged vegetation forms a vertical mixing layer that promotes mass and momentum transfer in the depth direction (see Nepf and Vivoni, ${ }^{12}$ Wilson et al.,${ }^{13}$ Poggi et al.,${ }^{14}$ Carollo et al.,${ }^{15}$ Ghisalberti and Nepf, ${ }^{16}$ Nepf et al., ${ }^{17}$ Nezu and Sanjou, ${ }^{18}$ Sukhodolov and Sukhodolova, ${ }^{19}$ and Okamoto et $a .^{20}$ ). This fact implies that the SR motion related to the interfacial gas transfer is quite different between the smooth-bottom and the roughness-bottom conditions even if the free-surface velocity is just the same.

Although the contribution of turbulence to gas transfer has been recognized, obtaining accurate turbulence data from field measurements in actual rivers is a difficult task. For practical purposes, the gas transfer velocity is generally evaluated by the bottom slope, bulk mean velocity, and water depth. Hence, in previous empirical formulae, contributions from turbulence have not been considered sufficiently. In contrast, the SD model deals directly with the surface divergence promoted by turbulence motions, and it is expected to fill the gap between a practical formula and scientific knowledge in open-channel flows.

In the 1990s, researchers developed PIV techniques for measuring surface currents in rivers, enabling the evaluation of vorticity and the diverse horizontal currents. Once these data are obtained, we can calculate gas transfer rates in natural rivers. The SD model was applied to various kinds of flow fields, e.g., (1) wind-induced currents ${ }^{21,22}$ (2) openchannel flow, ${ }^{3,5,23}$ and (3) oscillation tank. ${ }^{24-27}$ Note that the original SD model focuses mainly on the viscous small-scale motion, and it is questionable to use in large-scale openchannel turbulence such as natural rivers. In the first half of the present study, we develop an SD-based model included with effects of depth-scale motions for open-channel flows with a smooth bottom. In the second half, laboratory measurements described where we changed the bulk-mean velocity and water depth systematically to reveal the relation between surface divergence intensity and interfacial gas transfer. The result is that the coefficient of proportionality of the original SD model depends on the water depth. In contrast, the present modified SD model accounts for the effects of depth-scale motion on gas transfer and obtained much better fitting results than the original model. As the modified SD model covers both large and small-scale motions, it is useful over a wide range of hydraulic conditions, and consequently, leads to an improvement in the accurate prediction of the gas transfer velocity in open-channel flows.

\section{THEORETICAL BACKGROUND}

McCready et $a l .^{2}$ linearized the transport equation of dissolved gas concentration using the scaling relationship

$$
k_{L}^{+} \sim \sqrt{\frac{\beta_{+}^{\prime}}{S}},
$$

where $k_{L}^{+}=k_{L} / U_{c}$ and $\beta^{\prime+}=\beta^{\prime} T_{c}, S \equiv v / D$ is the Schmidt number, $D$ is the diffusion constants of dissolved oxygen, and $v$ is the kinematic viscosity. $U_{c}$ and $T_{c}$ are the characteristic velocity and time, respectively. Applying the friction velocity, $U_{*}$ and $v / U_{*}^{2}$, to the velocity and time scales in the following viscous scaling forms,

$$
\begin{aligned}
U_{c} & \equiv U_{*}, \\
T_{c} & \equiv v / U_{*}^{2}, \\
L_{c} & \equiv U_{c} T_{c}=v / U_{*},
\end{aligned}
$$

we obtain the popular scaling relation

$$
k_{L} \sim \sqrt{D \beta^{\prime}}
$$

which appears in the original SD model and assists in evaluating the gas transfer velocity from the surface velocity divergence. In open-channel flows, maximum vortex development is restricted by the finite water depth. Because the SD model cannot directly consider the effect of integral scales, a coefficient of proportionality depends on the water depth (see Section IV D). This implies that it may be difficult to evaluate the gas transfer velocity using only the viscous scales of Eq. (2). Therefore, the contribution to gas transfer from other scale events much larger than the viscous motion should also be considered. The present study highlights two kinds of vortex scales: one a small scale vortex related to viscous wall friction and the other a large scale vortex such as water depth size associated with secondary currents. 
The turbulence dissipation rate $\varepsilon$ is introduced to connect these difference scales. The definition of dissipation rate gives the scaling relation

$$
\varepsilon \sim v \frac{U_{c}^{2}}{L_{c}^{2}}\left(=v \frac{U_{*}^{2}}{L_{c}^{2}}\right) .
$$

In contrast, as explained by Tennekes and Lumley ${ }^{29}$ the dissipation rate in regard to large-scale motion is scaled in the manner

$$
\varepsilon \sim \frac{U_{H}^{3}}{L_{H}},
$$

in which $U_{H}$ and $L_{H}$ are the characteristic velocity and length of the depth-scale vortex. As cascade process guarantees that the turbulence dissipation rate is constant, the correspondence

$$
v \frac{U_{c}^{2}}{L_{c}^{2}} \sim \frac{U_{H}^{3}}{L_{H}} \rightarrow \frac{U_{c}}{L_{c}} \sim\left(\frac{U_{H}^{3}}{v L_{H}}\right)^{1 / 2}
$$

holds. Eq. (1) leads to

$$
k_{L} / U_{c}=k_{L} / U_{*} \sim \sqrt{\beta^{\prime} T_{c} / S}=\sqrt{\left(\beta^{\prime} L_{c} / U_{c}\right) / S} .
$$

Substituting in Eq. (6) yields

$$
\frac{k_{L}}{U_{*}} \sim \sqrt{\frac{\beta^{\prime}}{S}\left(\frac{v L_{H}}{U_{H}^{3}}\right)^{1 / 2}}, k_{L} \sim \sqrt{\frac{\beta^{\prime} v}{S}\left(\frac{U_{*}^{2} L_{H}^{1 / 2}}{v^{1 / 2} U_{H}^{3 / 2}}\right)} \sim \sqrt{L^{+} \beta^{\prime} D},
$$

in which

$$
L^{+}=\left(\frac{U_{*}^{2} L_{H}^{1 / 2}}{v^{1 / 2} U_{H}^{3 / 2}}\right)
$$

Eq. (9) leads to

$$
\begin{aligned}
L^{+} & =\left(\frac{U_{*}^{2} L_{H}^{1 / 2}}{v^{1 / 2} U_{H}^{3 / 2}}\right)=\frac{U_{*}^{2} L_{H}^{1 / 2} \times U_{H}^{1 / 2}}{v^{1 / 2} U_{H}^{3 / 2} \times U_{H}^{1 / 2}}=\frac{U_{*}^{2} L_{H}^{1 / 2} U_{H}^{1 / 2}}{U_{H}^{2} v^{1 / 2}} \\
& =\left(\frac{U_{*}}{U_{H}}\right)^{2} \operatorname{Re}^{1 / 2}=C_{v}{ }^{2} \mathrm{Re}^{1 / 2},
\end{aligned}
$$

in which $C_{v} \equiv U_{*} / U_{H}$ means friction velocity normalized by large-scale characteristic velocity and $\operatorname{Re} \equiv U_{H} L_{H} / v$ is the macroscale Reynolds number. This suggests that $L^{+}$increases as the Reynolds number and the bottom friction become large. That is to say, it implies that the bottom fiction promotes significantly the interfacial gas transfer. The bottom-oriented larger-scale coherent vortice motion such as bursting events is generated in roughness open-channel flows such as submerged vegetation channel than in flat bottom open-channel flows, and they are expected to increase the surface renewal rate, i.e., effective interfacial gas transfer. $L^{+}$expressed by Eq. (10) is found to correspond to this mechanism.

The free-surface velocity and water depth are chosen as the large-scale characteristic velocity and length in the manner

$$
\begin{aligned}
U_{H} & \sim U_{s}, \\
L_{H} & \sim H,
\end{aligned}
$$

and result in a new formula for the gas transfer velocity using surface divergence,

$$
k_{L} \sim \sqrt{L^{+} D \beta^{\prime}}, \quad L^{+}=\left(\frac{U_{*}^{2} H^{1 / 2}}{v^{1 / 2} U_{s}^{3 / 2}}\right),
$$

which we validate using measured data of the gas transfer velocity and turbulence statistics. Its advantage over the original SD model devised by McCready et al. ${ }^{2}$ is demonstrated in Section IV D.

It is very important to find hydrodynamic parameters that govern the surface velocity divergence because accurate SD measurements are generally difficult to take in field surveys. Hence we focus on $\beta^{\prime} ; U_{s}$ can be measured by the present surface PIV system. Additionally, water depth, $H$, water density, $\rho$, and viscosity, $\mu$, are also considered for dimensional analysis. The $\Pi$-theorem provides normalized numbers,

$$
\begin{gathered}
\Pi_{\beta^{\prime}}=v \beta^{\prime} / U_{s}^{2}, \text { where } v \equiv \mu / \rho, \\
\Pi_{H}=U_{s} H / v=\operatorname{Re}_{s},
\end{gathered}
$$

in which $\operatorname{Re}_{s}$ is a $U_{s}$-based Reynolds number. Therefore, the surface divergence intensity normalized by the surface velocity and kinematic viscosity is expected to depend on the Reynolds number as follows:

$$
\frac{v \beta^{\prime}}{U_{s}^{2}} \sim \text { func }_{1}\left(\operatorname{Re}_{s}\right)
$$

in which func $_{1}$ is a function of $U_{s}$-based Reynolds number.

When $U_{s}$ is replaced by $U_{*}$ in the left-side term of Eq. (15), the non-dimensional number becomes $\beta^{\prime+}$ used in Eq. (1), and following relation can be assumed:

$$
\beta^{\prime} \equiv \frac{v \beta^{\prime}}{U_{*}^{2}} \sim \text { func }_{2}\left(\operatorname{Re}_{s}\right)
$$

in which func 2 is a function of $U_{s}$-based Reynolds number. For practical modeling of the SR rate, we must select only those hydrodynamic values obtained using the HPIV (horizontal PIV) measurements. It is difficult to measure $U_{*}$ accurately for natural rivers and Eq. (12) is lacking in practical utility. Therefore, it hoped that $U_{*}$ is expressible by some other velocity scale which can be measured in field surveys, e.g., the surface velocity $U_{s}$. When we know the function form func 2 in Eq. (16), the gas transfer velocity given by Eq. (12) can be evaluated without information of the friction velocity. The functions, $f u n c_{1}$ and $f u n c_{2}$, are defined by fitting operation using measured data as mentioned in Section IV B 2.

\section{MEASUREMENT PROCEDURE}

\section{A. Experimental setup}

Figure 2 depicts the tilted glass flume used in this study. $B$ and $H$ in the figure denote the channel width and water depth, respectively. The experimental flume is $16 \mathrm{~m}$ long, $40 \mathrm{~cm}$ wide, and $50 \mathrm{~cm}$ high. The water current through the flume was controlled by a computer and regulated using an electromagnetic flow meter. The streamwise, vertical, and spanwise coordinates are denoted $x, y$, and $z$, respectively. The vertical origin, $y=0$ is set at the channel bed. The time-averaged velocity components in the $x, y$, and $z$ directions are defined 


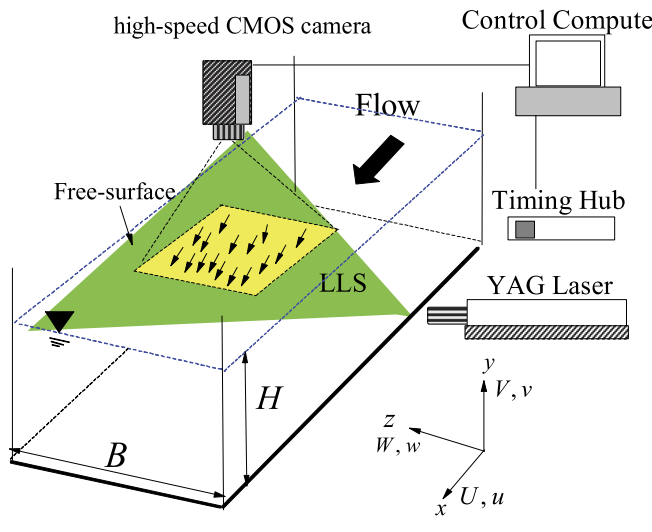

(a) PIV system for velocity measurement

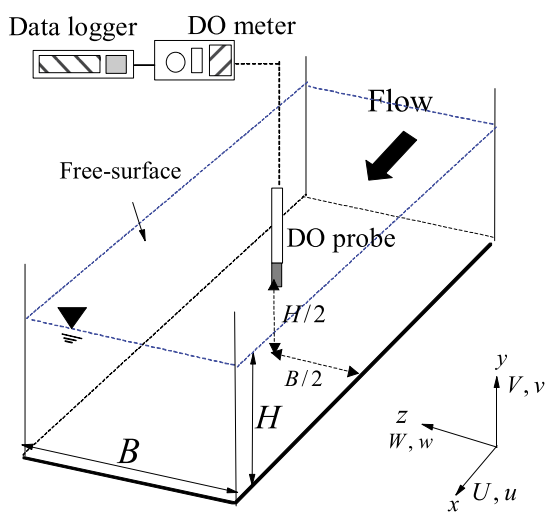

(b) DO measurement system
FIG. 2. Experimental setup for velocity measurement: (a) PIV system for velocity measurement in the free-surface, (b) DO measurement system to obtain interfacial gas transfer velocity. $B$ is the channel width, $H$ is the water depth, and LLS means laser light sheet for projection of measurement field. The origin of $y$-axis is situated at the flume bottom. as $U, V$, and $W$, respectively, and the corresponding turbulence fluctuations are $u, v$, and $w$. The turbulence intensities, calculated using the root-mean-square procedure, are $u^{\prime}, v^{\prime}$, and $w^{\prime}$.

The laser light sheet (LLS) was illuminated using a 2-W YAG continuous laser (Fig. 2(a)). Images were acquired on two size scales. The S-HPIV (small-scale horizontal PIV) obtained highly accurate measurements of the SD in the centerline of the flume, covering an image area of $(9 \times 9) \mathrm{cm}^{2}$ with an interrogation window size of $(3.3 \times 3.3) \mathrm{mm}^{2}$. The other imaging approach, L-HPIV (large-scale horizontal PIV), focused on the spanwise distribution of the fluid motion, covering a larger image area of $(30 \times 30) \mathrm{cm}^{2}$ with an interrogation window size of $(7.5 \times 7.5) \mathrm{mm}^{2}$. For the HPIV measurements, the $2-\mathrm{mm}$ thick LLS was horizontally projected just $1 \mathrm{~mm}$ below the interface because clear images could not be acquired at the interface elevation.

Calmet and Magnaudet ${ }^{23}$ calculated the vertical profiles of velocity divergence near the free surface from a large-eddy simulation (LES). They designated a near-constant divergence intensity below $y^{\prime+} \approx 12$, where $y^{\prime}$ is the distance from the freesurface in the direction of the flume bottom and $y^{\prime+} \equiv y^{\prime} U_{*} / v$ is the corresponding normalized value. In the present study, the LLS elevation range, i.e., $1 \mathrm{~mm}$ under the free surface corresponds to $5.2<y^{\prime+}<16.2$, suggesting that the measured SD almost equals that on the free surface. Because of the free-slip condition, the velocity profiles exhibit much smaller vertical variations near the free surface than the dissolved gas concentration. The laser Doppler anemometer (LDA; Dantec, Inc.) is able to provide also highly accurate velocity measurements in the vertical direction. In the hydraulic case with $U_{m}=20 \mathrm{~cm} / \mathrm{s}$ and $H=8 \mathrm{~cm}$, the LDA scans from 0.2 to $1.2 \mathrm{~mm}$ beneath the free surface at $0.2-\mathrm{mm}$ intervals. Standard deviations of the vertical profiles of $U(y)$ and $u^{\prime}(y)$ were 0.173 and $0.054 \mathrm{~cm} / \mathrm{s}$, respectively. These values are sufficiently small to assume a high correlation between the fluid at the LLS elevation and that at the free surface. Therefore, the velocity data collected at the LLS elevation can substitute for the velocity components at the free-surface.

Furthermore, to evaluate the friction velocity at the bottom, we conducted VPIV (vertical PIV) measurements in the $x-y$ plane, projecting the LLS along the flume centerline. The specific density and diameter of the tracer particles were 1.02 and $100 \mu \mathrm{m}$, respectively. The spatial and temporal distributions of the tracer patterns were taken using a high-speed CMOS camera situated over the free surface. The frame rate was $100-500 \mathrm{~Hz}$, depending on the bulkmean velocity. The frame rate corresponds to the time lag between two consecutive images and is used for calculating the two-dimensional velocity components. The sample rate, which specifies the time interval between the obtained velocity data, was given as $30 \mathrm{~Hz}$ by the function generator.

The gas transfer velocity was obtained from the DO measurements by the method described in Herlina and Jirka, ${ }^{28}$ in which the reaeration coefficient $k_{2}$ and convection velocity $k_{L} \equiv k_{2} H$ could be obtained through DO time-decay as mentioned in Section IV C. The measured transfer velocity was adjusted to that expected at a water temperature of $20^{\circ} \mathrm{C}$. In the present experiment, the probe of the DO meter was situated at a half-depth elevation in the channel centerline as shown in Fig. 2(b). DO gases were well-mixed through the baffle boards and returning pipe before flowing through the main flume. Furthermore, to maintain constant water temperature, we controlled the room temperature with five large air conditioners. After driving the motor pump for $2 \mathrm{~h}$, the system was reaerated to prevent any influence from heat generated by the pump unit. The measured DO signals were transferred to the data logger at $1.0 \mathrm{~min}$ intervals.

\section{B. Hydraulic condition}

Hydraulic conditions are listed in Table I, in which $U_{m}$ is the bulk-mean velocity and $U_{s}$ is the time-averaged freesurface streamwise velocity at the centerline of the flume. Fr and Re are the Froude and Reynolds numbers, respectively, defined as Fr $\equiv U_{m} / \sqrt{g H}$ and $\operatorname{Re} \equiv U_{m} H / v$. As the DO measurements are time consuming (10-40 h), the room and water temperatures were maintained constant using air conditioners. The fifteen hydraulic cases were selected by systematically varying $U_{m}$ and $H$. Nezu and Nakagawa ${ }^{7}$ suggested that secondary currents exert a remarkable influence in openchannel flows at aspect ratios $B / H$ smaller than 5.0. In the present smooth-bed experiments, the water depth was chosen to examine the effects of secondary currents on gas transfer flux. 
TABLE I. Hydraulic condition for the smooth bottom cases. $U_{m}$ is the bulk-mean velocity, $U_{s}$ is the free-surface mean velocity, $H$ is the water depth, $B / H$ is the aspect ratio, $\beta^{\prime}$ is the divergence intensity, $k_{L}$ the is gas transfer velocity, $\operatorname{Re}=U_{m} H / v$ is the macroscale Reynolds number, and $\mathrm{Fr}=U_{m} / \sqrt{g H}$ is the Froude number. The water depth and the bulk-mean velocity are systematically varied.

\begin{tabular}{lccccccrc}
\hline \hline CASE & $U_{m}(\mathrm{~cm} / \mathrm{s})$ & $U_{s}(\mathrm{~cm} / \mathrm{s})$ & $H(\mathrm{~cm})$ & $B / H$ & $\beta^{\prime}(1 / \mathrm{s})$ & $k_{L}(\mathrm{~cm} / \mathrm{s}) \times 10^{-3}$ & Re & Fr \\
\hline U10H6 & 10.0 & 12.7 & & & 0.74 & 0.24 & 6000 & 0.13 \\
U20H6 & 20.0 & 23.6 & 6.0 & 6.7 & 1.35 & 0.52 & 12000 & 0.26 \\
U30H6 & 30.0 & 36.1 & & & 2.31 & 0.66 & 18000 & 0.39 \\
U10H8 & 10.0 & 13.0 & & & 0.60 & 0.35 & 8000 & 0.11 \\
U20H8 & 20.0 & 24.9 & 8.0 & 5.0 & 1.37 & 0.69 & 16000 & 0.23 \\
U30H8 & 30.0 & 37.1 & & & 2.31 & 0.82 & 24000 & 0.34 \\
U10H10 & 10.0 & 12.0 & & & 0.53 & 0.44 & 10000 & 0.10 \\
U20H10 & 20.0 & 23.7 & 10.0 & 4.0 & 1.20 & 0.77 & 20000 & 0.20 \\
U30H10 & 30.0 & 35.5 & & & 2.30 & 0.88 & 30000 & 0.30 \\
U10H15 & 10.0 & 12.1 & & & 0.68 & 0.56 & 15000 & 0.08 \\
U20H15 & 20.0 & 23.8 & 15.0 & 2.7 & 1.19 & 0.99 & 30000 & 0.16 \\
U30H15 & 30.0 & 36.5 & & & 2.39 & 1.25 & 45000 & 0.25 \\
U10H20 & 10.0 & 11.9 & & & 0.46 & 0.61 & 20000 & 0.07 \\
U20H20 & 20.0 & 23.7 & 20.0 & 2.0 & 1.29 & 1.19 & 40000 & 0.14 \\
U30H20 & 30.0 & 36.0 & & & 2.58 & 1.70 & 60000 & 0.21 \\
\hline \hline
\end{tabular}

The measured region was approximately $12 \mathrm{~m}$ downstream from the channel entrance, where the turbulent flow was fully developed. The measurement should be conducted downstream of the region where the boundary layer is fully developed. Kirlkgoz and Ardichoglu ${ }^{30}$ proposed the following useful formula about the developing distance $x_{d}$ in the open-channel flows:

$$
x_{d} / H=76-0.0001 \mathrm{Re} / \mathrm{Fr} .
$$

Normalized developing distance $x_{d} / H$ can be evaluated by a function of ratio of Re to Fr. It is generally known that a larger depth condition requires a longer developing distance of the boundary layer. In our maximum depth case $(H$ $=20 \mathrm{~cm}$ ), this formula results in the developing distance is $x_{d}=9.6 \mathrm{~m}(<12 \mathrm{~m})$, and it concludes that the present measured section is positioned downstream of the developing region.

\section{RESULTS AND DISCUSSION}

\section{A. Mean flow field}

\section{Spanwise variation of horizontal velocity components}

From the L-HPIV measurements, we can understand the whole-scale flow motion and obtain the spanwise profile of the lateral velocity at the free surface. In these measurements, we varied the water depth $H$ at settings 6,10 , and $20 \mathrm{~cm}$. Figure 3 shows a spanwise profile of time-averaged velocity $W(\mathrm{z})$, in which the previous result of experiment (Nezu and $\left.\operatorname{Rodi}^{31}\right)$ is included for $H=20 \mathrm{~cm}(B / H=2.0)$. In all instances, the velocities are negative near the right side wall $(z /(0.5 B)=1)$, indicating that part of the secondary current converges toward the centerline of the channel. This property significantly depends on the water depth. In shallow water setting $(H=6 \mathrm{~cm})$, the spanwise motion seems to be limited to $10 \mathrm{~cm}(z /(0.5 B)=0.5)$ near the sidewall, and the spanwise velocity is almost zero at short distances from the sidewall. Interestingly, the negative distribution extends to the centerline for $H=10 \mathrm{~cm}$. In the deepest water case $(H$ $=20 \mathrm{~cm}$ ), the spanwise velocity reverses direction near the flume centerline, implying strong lateral flows over the whole width. Although the spanwise velocity increases with increasing streamwise velocity, the formation of secondary currents at the free surface is independent of the bulk-mean velocity. The diameters of two vortices created near each side-wall are equal to the water depth in the case $(H=20 \mathrm{~cm}, B / H$ $=2.0$ ), and this implies that vortices meet each other. In such a status, cross-sectional mass transfer is more significantly accelerated than in the condition in which only smaller-scale secondary currents are generated. The same feature could be found in the previous experimental data. ${ }^{31}$ Although the sign of $W$ changes near the mid-width, zero velocity $(W=0)$ position is not observed at the centerline in the present result. The imaging area of the present L-HPIV does not cover the whole width region. It focused mainly on the one-side halfwidth region because we hold the resolution of the image as high as possible. Therefore, the lateral center of the imaging is not consistent with the centerline of the channel. Such a photographing method may induce a non-symmetrical profile of $W$.

Spanwise profiles of the time-averaged streamwise velocity $U(\mathrm{z})$ (Fig. 4) exhibit in all cases a single peak near the centerline of the channel. As the aspect ratio diminishes, the spanwise gradient of the streamwise velocity becomes larger because the side-wall vortices meet and accumulate momentum near the centerline. For the $B / H=2.0$ case, the present experiment could reproduce the results of previous experiment reported by Nezu and Rodi ${ }^{31}$ and the Reynolds stress model calculated by Khang and Choi. ${ }^{32}$ From the principle of fluid continuity, downward and upward flows should be locally generated in the centerline and the sidewall region, respectively, as shown in Fig. 1. We conjecture that such depth-scale circulation vertically expedites the 


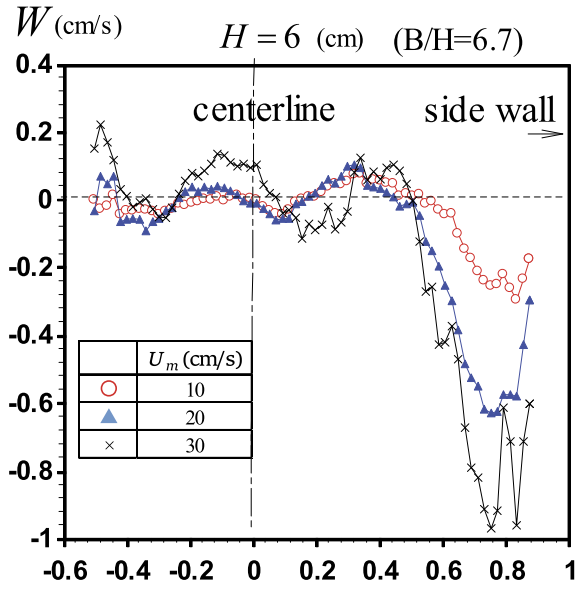

(a)

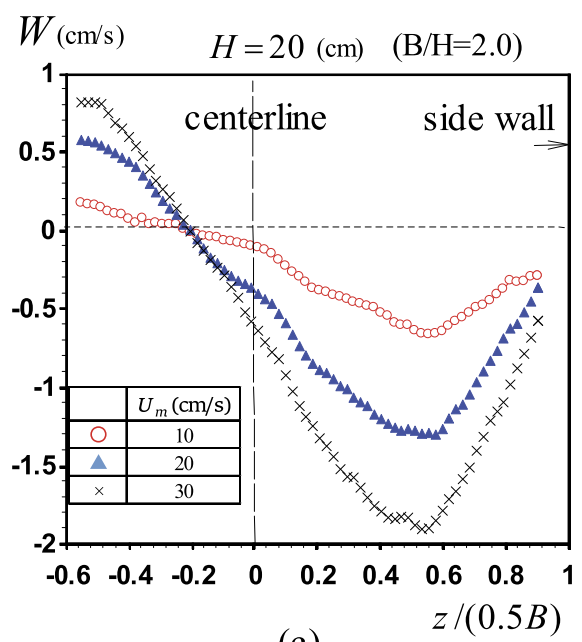

(c)

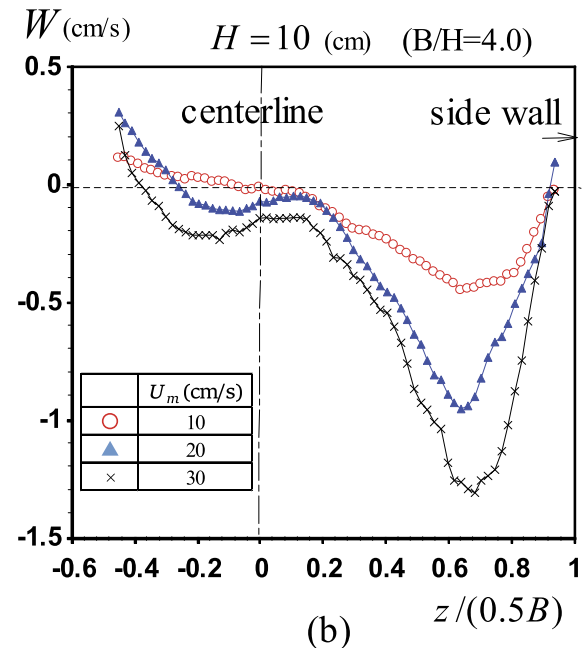

(b)

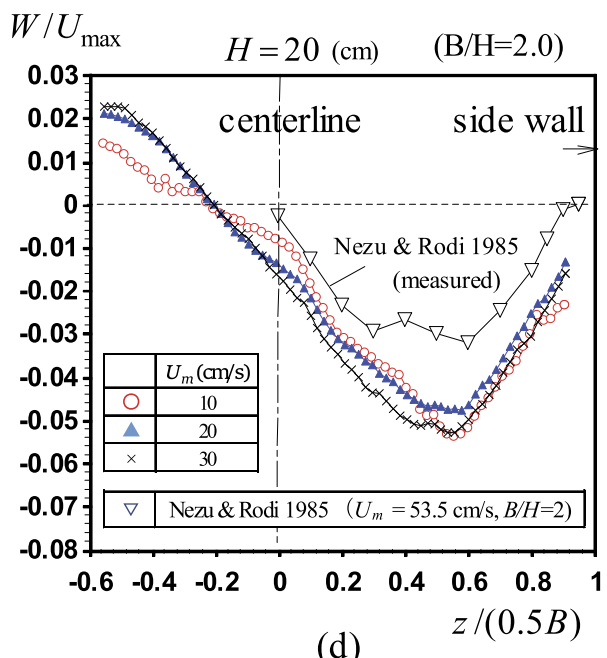

FIG. 3. Lateral profiles of timeaveraged spanwise velocity with comparison of different water depth cases: (a) $H=6 \mathrm{~cm}$, (b) $H=10 \mathrm{~cm}$, (c) $H=20 \mathrm{~cm}$, and (d) comparison with previous experimental data by Nezu and Rodi. ${ }^{31}$ There are significant spanwise currents toward the centerline for the $H=20 \mathrm{~cm}(B / H=2.0)$. dissolved gas transport. The original SD model admits no contribution from secondary currents because it considers only the SD.

\section{Vertical profile of streamwise velocity}

Figure 5 shows vertical profiles of the time-averaged streamwise velocities at $U_{m}=10$ and $30 \mathrm{~cm} / \mathrm{s}$, obtained by VPIV (Vertical PIV) measurements. The target spanwise position is the flume centerline $(z / B=0)$. The bottom shear stress establishes a typical boundary layer and reduces significantly the near-bottom velocity. Of particular significance is the velocity dip (i.e., elevation drop of the maximum velocity) at an aspect ratio of $B / H=2.0$. This occurs because the two secondary currents (side-wall vortices) meet in the centerline and disturb the boundary layer from the bottom (see Fig. 1), as reported by Nezu and Nakagawa. ${ }^{7}$ Influences of secondary currents on the velocity profile are independent of the velocity range of the present experiment. Furthermore, the maximum velocity appears at $y / H=0.6-0.7$ for the $B / H$ $=2.0$, irrespectively of the mean velocity. This significant tendency could be observed in both the present study and previous study by Nezu and Rodi. ${ }^{31}$ Although the present PIV measurements give only fragmentary information, typical secondary currents undoubtedly form in the rectangular channel.

\section{B. Properties of surface velocity divergence}

\section{Instantaneous horizontal velocity field}

Instantaneous surface velocity divergence at the airwater interface $\tilde{\beta}$ (where the tilde indicates instantaneous components) is defined as

$$
\tilde{\beta} \equiv \frac{\partial \tilde{u}}{\partial x}+\frac{\partial \tilde{w}}{\partial z}=-\frac{\partial \tilde{v}}{\partial y} .
$$

Here, the coordinate system is relative to the water surface, and thus the instantaneous vertical velocity is always zero at the free-surface. Using Eq. (18) to substitute for $\tilde{v}$, the transport equation of the instantaneous dissolved gas concentration $\tilde{c}$ becomes

$$
\frac{\partial \tilde{c}}{\partial t}+\tilde{u} \frac{\partial \tilde{c}}{\partial x}+\tilde{w} \frac{\partial \tilde{c}}{\partial z}-\tilde{\beta} y \frac{\partial \tilde{c}}{\partial y}=D \frac{\partial^{2} \tilde{c}}{\partial y^{2}} .
$$

Note that the axis of $x, z$, and $y$ in Eqs. (18) and (19) are relative to the free surface. Hence, they are not parallel and vertical to the bottom plain, when the free surface significantly varies in space and time. From this expression, Tamburrino and Gulliver ${ }^{3}$ deduced that $\tilde{\beta}$ is directly related to the interfacial mass transfer because spanwise and streamwise variations of concentration are much smaller than vertical one. 


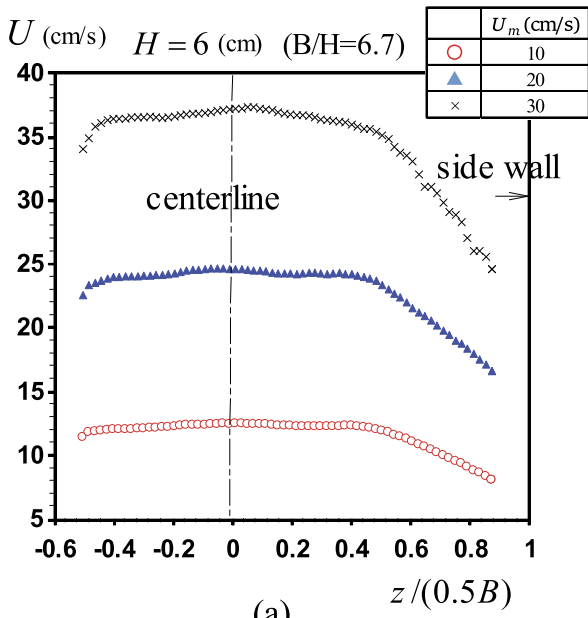

(a)

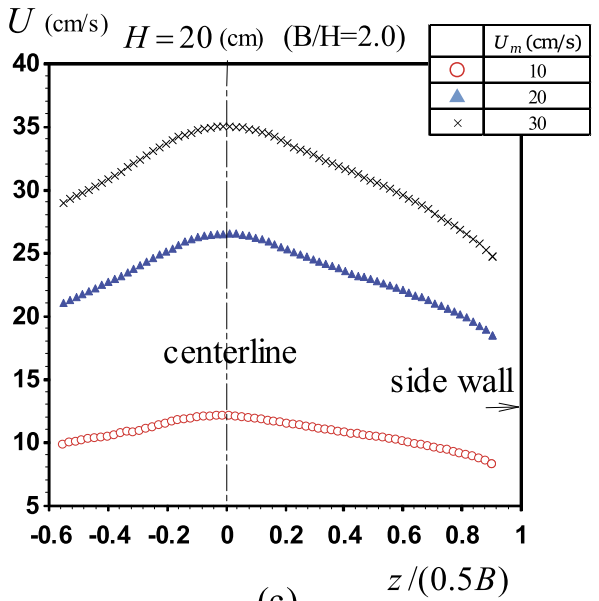

(c)

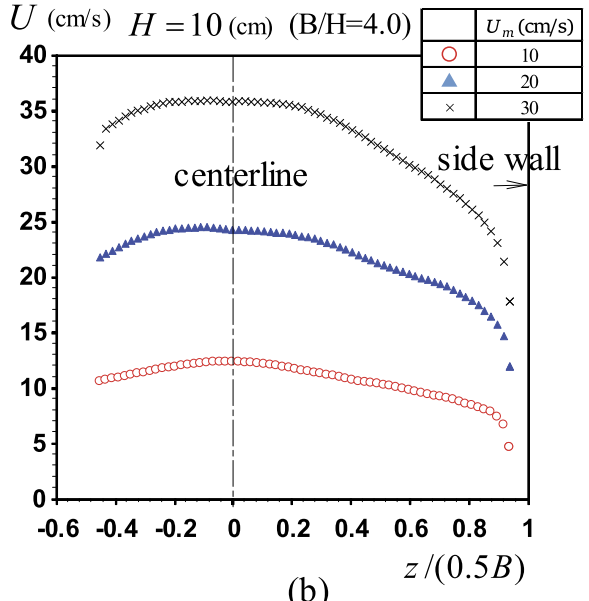

(b)

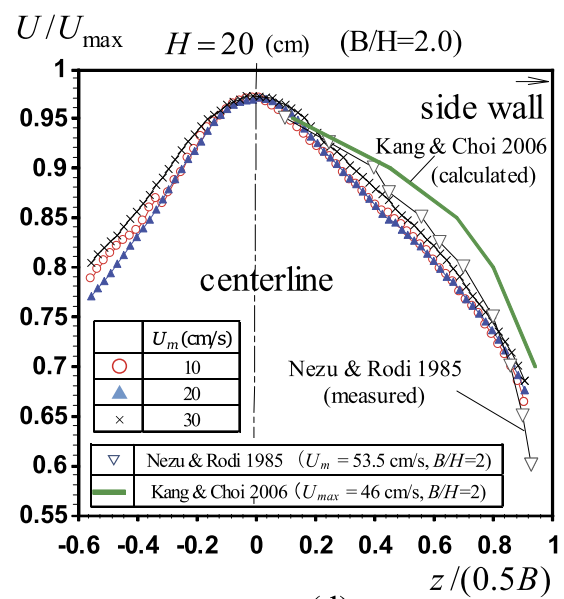

(d)
FIG. 4. Lateral profiles of timeaveraged streamwise velocity with comparison of different water depth cases: (a) $H=6 \mathrm{~cm}$, (b) $H=10 \mathrm{~cm}$, (c) $H=20 \mathrm{~cm}$, and (d) comparison with previous experimental data ${ }^{31}$ and numerical data. ${ }^{32}$ The gradient of $U$ $(z)$ is the most remarkable for the $H$ $=20 \mathrm{~cm}(B / H=2.0)$ because of meeting of side-wall vortices.
Figure 6 shows for $U_{s}=12.3 \mathrm{~cm} / \mathrm{s}$ and $H=8 \mathrm{~cm}$ the instantaneous horizontal distribution of $\tilde{\beta}$ and the velocity vectors $\left(\tilde{u}-\tilde{u}_{r}, \tilde{w}-\tilde{w}_{r}\right) .\left(\tilde{u}_{r}, \tilde{w}_{r}\right)$ is a reference velocity vector. We focused on a specific local point with large divergence (convergence), and defined the reference velocity vector as the velocity at this location. The reference velocity components are subtracted from the original velocity vector distribution $(\tilde{u}, \tilde{w})$. These profiles were acquired as a time series at 0.033 -s intervals. In the convergence zone $\tilde{\beta}<0$, the surrounding momentum concentrates and a downward current is generated as indicated in Fig. 1. In contrast, in the divergence zone $\tilde{\beta}>0$, the momentum diverges as an upward current flows. A converging zone marked $\mathrm{C} 1$ corresponding negative divergence is traveling downstream during $0.033 \mathrm{~s}$ (Figures 6(a) and 6(b)). Surrounding fluids are found to come close to this zone, and the sign of instantaneous divergence implies that the downward currents are accompanied there. At $t=0.033 \mathrm{~s}$, a new convergence zone marked $\mathrm{C} 2$ appears upstream in the measurement area. Figure 6(c) focuses on C2, in which the reference velocity corresponds to the velocity at

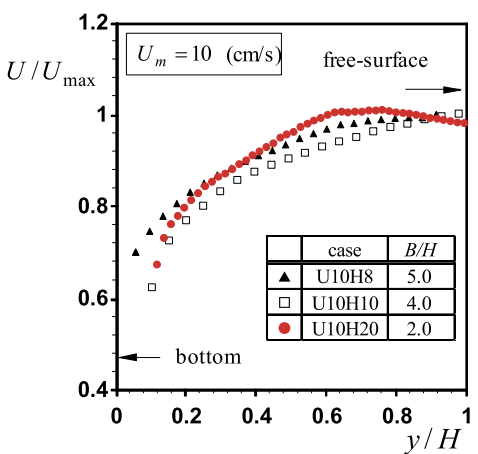

(a)

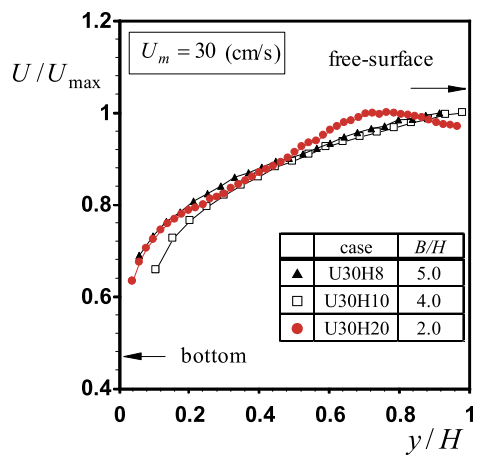

(b)

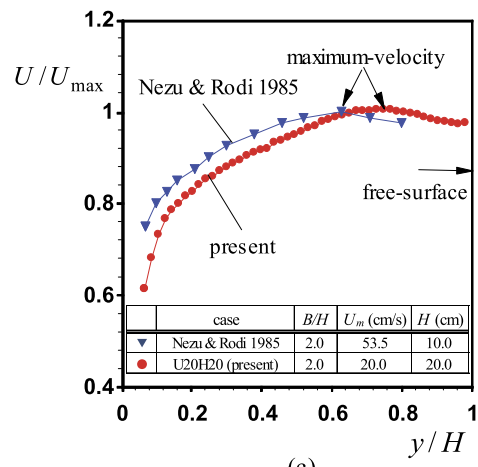

(c)

FIG. 5. Vertical profiles of time-averaged streamwise velocity: (a) $U_{m}=10 \mathrm{~cm} / \mathrm{s}$, (b) $U_{m}=30 \mathrm{~cm} / \mathrm{s}$, (c) comparison with previous data by Nezu and Rode ${ }^{31}$ for $B / H=2$, Influence of secondary currents on the velocity profile is independent of the velocity range of the present experiment. The maximum velocity appears at $y / H=0.6-0.7$ for the $B / H=2.0$, irrespectively of the mean velocity. 
(a) $t=0 \mathrm{~s}$ for $\mathrm{C} 1$

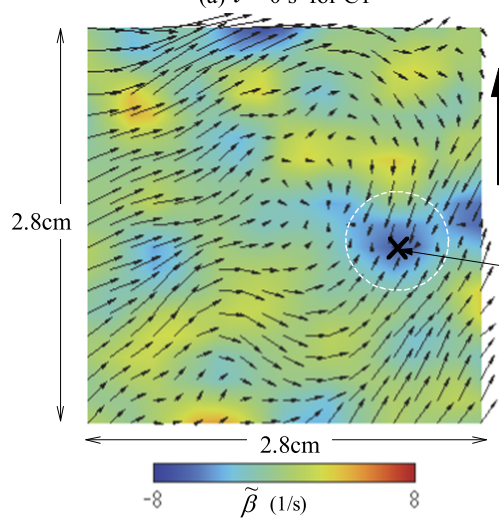

(d) $t=0.033 \mathrm{~s}$ for $\mathrm{D} 1$

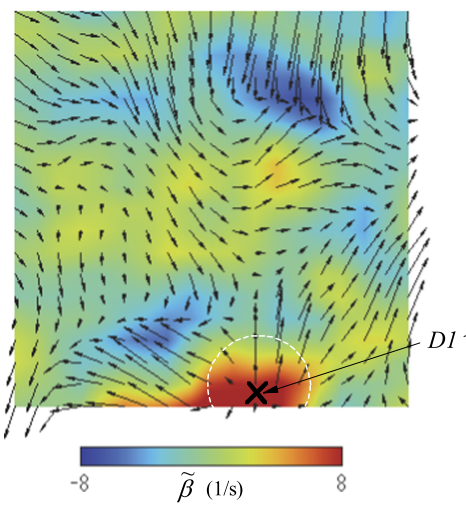

(b) $t=0.033 \mathrm{~s}$ for $\mathrm{C} 1$

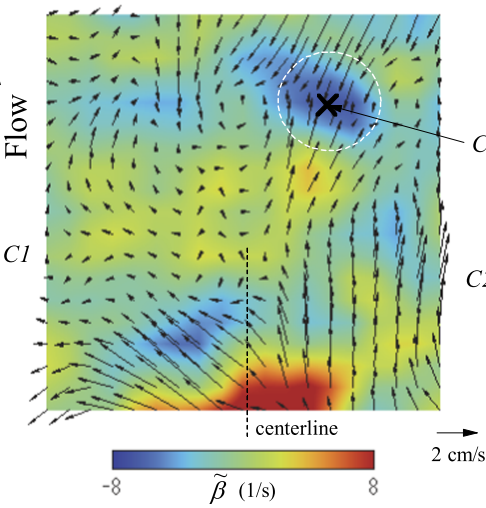

(e) $t=0.067 \mathrm{~s}$ for $\mathrm{D} 1$

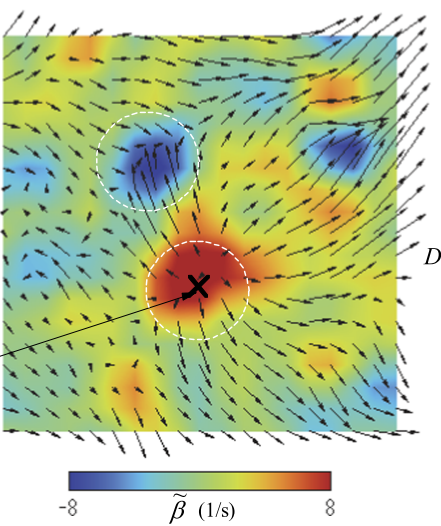

(c) $t=0.033 \mathrm{~s}$ for $\mathrm{C} 2$

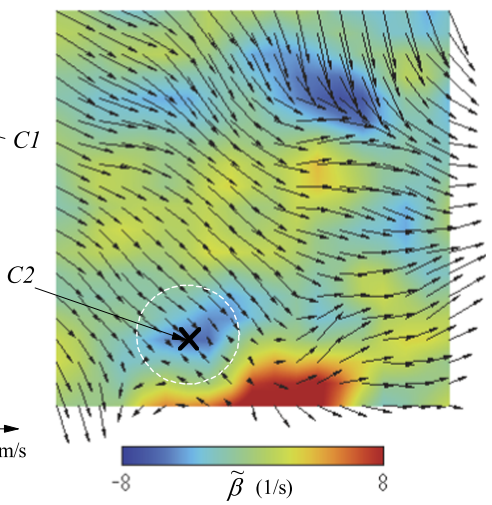

(f) $t=0.100 \mathrm{~s}$ for $\mathrm{D} 1$

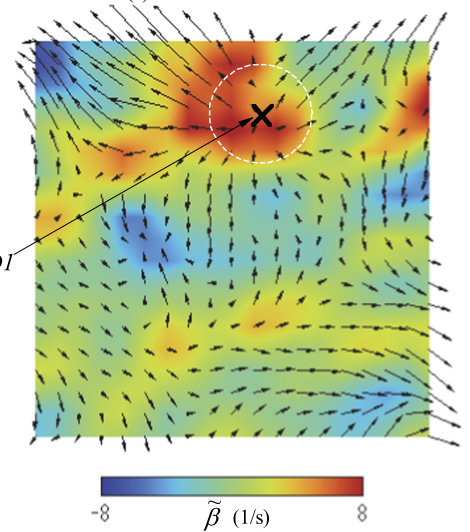

FIG. 6. Time-variation of horizontal distribution of surface velocity divergence and horizontal velocity vectors $\left(\tilde{u}-\tilde{u}_{r}, \tilde{w}-\tilde{w}_{r}\right)$, every $0.033 \mathrm{~s}$ for the cases of U30H20. $\left(\tilde{u}_{r}, \tilde{w}_{r}\right)$ are reference velocity vectors corresponding to each specific zone with a large divergence value. The results for (b)-(d) are acquired at the same timing, $t=0.033 \mathrm{~s}$. (a) and (b) focus on first converging parcel marked $\mathrm{C} 1$, (c) focuses on second converging parcel marked $\mathrm{C} 2$, and (d)-(f) focuses on diverging parcel marked D1. They are traveling downstream together with large divergence values.

the position of $\mathrm{C} 2$. We can observe converging velocity vectors within the $\mathrm{C} 2$ area. Figures $6(\mathrm{~d})-6(\mathrm{f})$ indicate time series of streamwise traveling of divergence zone marked D1. The large positive divergence values match the specific zone where the velocity vectors locally spread.

\section{Relationship between surface velocity divergence and bulk mean velocity/water depth}

Figure 7 plots the SD intensities $\beta^{\prime}$ as a function of bulkmean velocity at various water depths. Similar to the turbulence intensity $\left(u^{\prime}, v^{\prime}, w^{\prime}\right), \beta^{\prime}$ equals the RMS of the instantaneous divergence of the surface velocity. $\beta^{\prime}$ was calculated from a time series of $\tilde{\beta}$ collected over $60 \mathrm{~s}$. This figure includes the previous data measured by Turney and Banerjee ${ }^{5}$ for comparison. This results in increase of $\beta^{\prime}$ quadratic-functionally corresponding to the increase of $U_{m}$ in the same manner as the previous data. The order of the present data is compatible with that of Turney and Banerjee. ${ }^{5}$ The present data imply that $\beta^{\prime}$ varies with the bulk-mean velocity and remains almost constant under the same bulk-mean velocity condition, irrespective of the water depth. Although $U_{m}$ has significant impacts on production of the surface divergence, this is a complicated mechanism related to the turbulence structure in the stream.

The turbulence generated near the bed is transferred toward the free-surface region, along with strong bursting events. Komori et al. ${ }^{10}$ demonstrated that the near-bottom and
SR eddy-induced bursting frequencies are strongly correlated. The coherent bursting motion diffuses significantly during its vertical transfer to the free surface. Figure 8 compares the turbulent kinetic energy in the free surface, $t_{k} \equiv\left(u^{\prime 2}+w^{2}\right) / 2$,

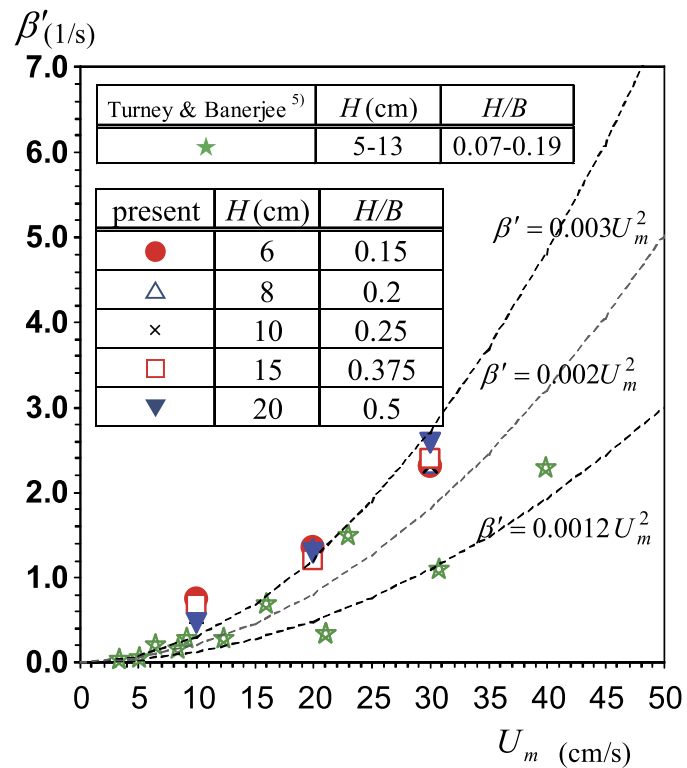

FIG. 7. Relation among divergence intensity, water depth, and surface velocity including result of previous study by Turney and Banerjee. ${ }^{5}$ Divergence intensity increases in quadratic form as $U_{m}$ becomes larger. 


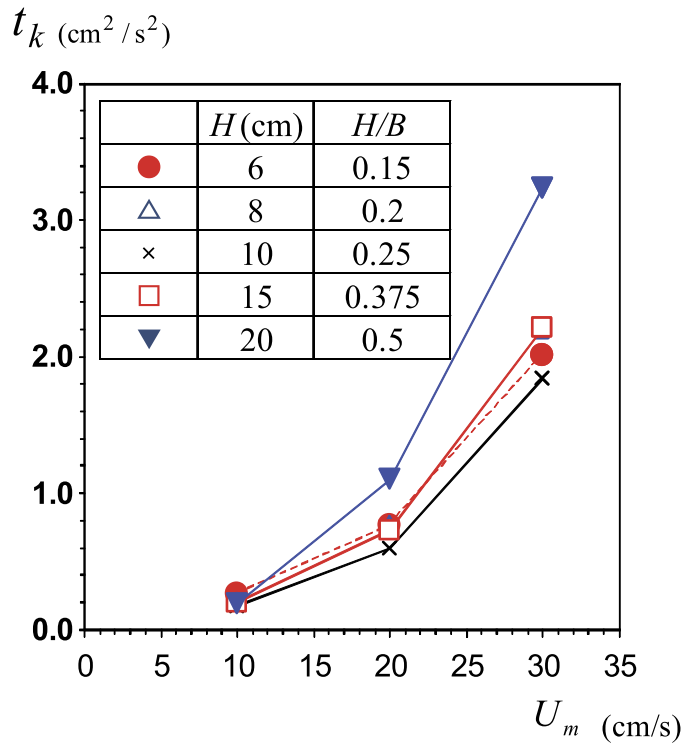

FIG. 8. Relation among turbulent kinetic energy, water depth, and surface velocity. Higher surface velocity induces larger bottom friction, and it results in larger turbulent kinetic energy in the free-surface.

for all cases. Turbulence is well known to be generated by wall friction in gravity-driven open-channel flows, and it is transported from the bottom toward to the free surface. In consequence, the free-surface turbulence has higher energy as the mean velocity becomes larger as indicated in this figure. Note that the turbulent kinetic energy increases slightly as the water depth becomes large for the U20 and U30 series. This is because not only bottom-oriented turbulence but also sidewall-oriented turbulence is transported toward the centerline in the free surface because of the secondary currents.

Direct measurements of the surface divergence are able to define the function forms, func $c_{1}$ and func $_{2}$, used in Eqs. (15) and (16). Figure 9 shows the relation between the normalized divergence $\Pi_{\beta^{\prime}}$ and the Reynolds number $\mathrm{Re}_{s}$, in which despite scattering being comparatively large at low Reynolds number, the fitting function

$$
v \beta^{\prime} / U_{s}^{2}=1.3 \times 10^{-3} \operatorname{Re}_{s}^{-0.392} \cong 1.3 \times 10^{-3} \operatorname{Re}_{s}^{-2 / 5}
$$

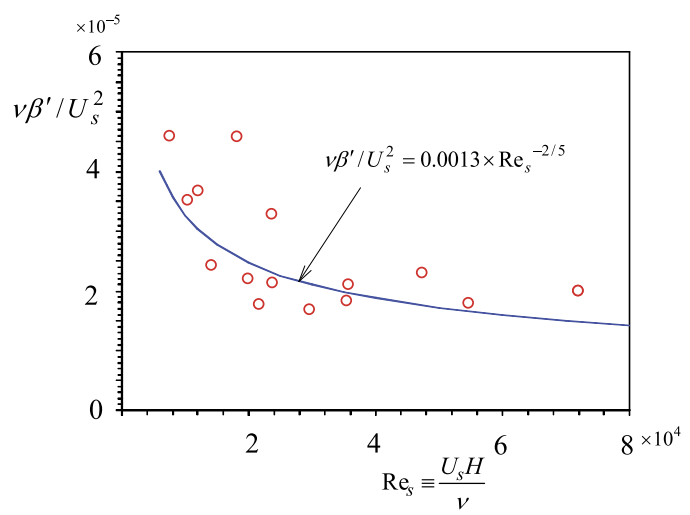

FIG. 9. Variation of surface divergence intensity normalized by the surface streamwise velocity with $U_{s}$-based Reynolds number. The surface divergence intensity is influenced by both the velocity and depth in the low Reynolds number regime, whereas normalized divergence intensity converges at high Reynolds regime, and it means that the divergence is not influenced by water depth. is observed, in which a coefficient of determination $R^{2}$ is 0.51 . This fitting curve was calculated using the least square method introducing a power approximation expression. The surface divergence is influenced by both the velocity and the water depth in the low Reynolds number regime. In contrast, $\Pi_{\beta^{\prime}}$ converges at high Reynolds number and hence the surface divergence is affected more significantly by velocity than by the water depth. In contrast, Figure 10 shows the relation between $\beta^{\prime+}$ and $\operatorname{Re}_{s}$, which suggests fitting function introducing the power approximation expression in the same manner as Eq. (20),

$\beta^{\prime+} \equiv v \beta^{\prime} / U_{*}^{2}=9.7 \times 10^{-2} \times \mathrm{Re}_{s}^{-0.197} \cong 9.7 \times 10^{-2} \mathrm{Re}_{s}^{-1 / 5}$.

The coefficient of determination $R^{2}$ is 0.21 . Eq. (21) yields the scaling relation

$$
U_{*}^{2} \sim \beta^{\prime} U_{s}^{1 / 5} H^{1 / 5} v^{4 / 5}
$$

\section{Properties of gas transfer velocity}

The mass transport equation of the normalized gas concentration $C^{\prime} \equiv\left(C_{s}-C\right) /\left(C_{s}-C_{0}\right)$ yields

$$
k_{2}=-\frac{d \ln C^{\prime}}{d t}
$$

where $C_{0}$ and $C_{s}$ are the initial and saturated gas concentrations, respectively. Figure 11 shows an example of the time-decay of a normalized gas concentration for $U_{m}=10$ $\mathrm{cm} / \mathrm{s}$ and $H=8 \mathrm{~cm}$, in which a period of linear decay occurs. We can evaluate the reaeration coefficient $k_{2}$ and convection velocity $k_{L}$ fitting the measured data to the linear relation, Eq. (23).

The relationship between the gas transfer velocity $k_{L}$ measured by DO sensor and the water depth is shown in Fig. 12. The gas transport across the interface is found to be enhanced corresponding to the bulk-mean velocity under the same depth condition. As the bottom friction velocity is generally proportional to the bulk-mean velocity, the present result can refer to a variation in the gas transfer velocity on the friction velocity and water depth. The boundary layer develops the whole depth region from the bottom to the free-surface in the open-channel flow. Nezu and Rodi, ${ }^{33}$ Nezu and Nakagawa ${ }^{7}$

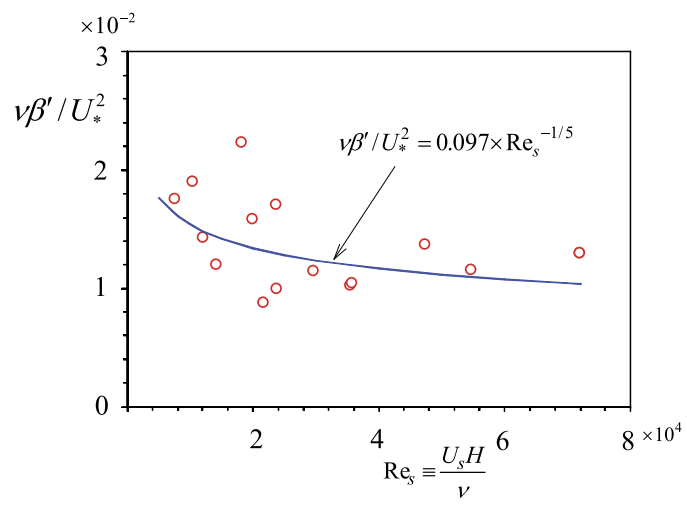

FIG. 10. Variation of surface divergence intensity normalized by a friction velocity with $U_{s}$-based Reynolds number. This fitting curve supports to express $U_{*}$ by Reynolds number $\operatorname{Re}_{s}$ and divergence intensity. 


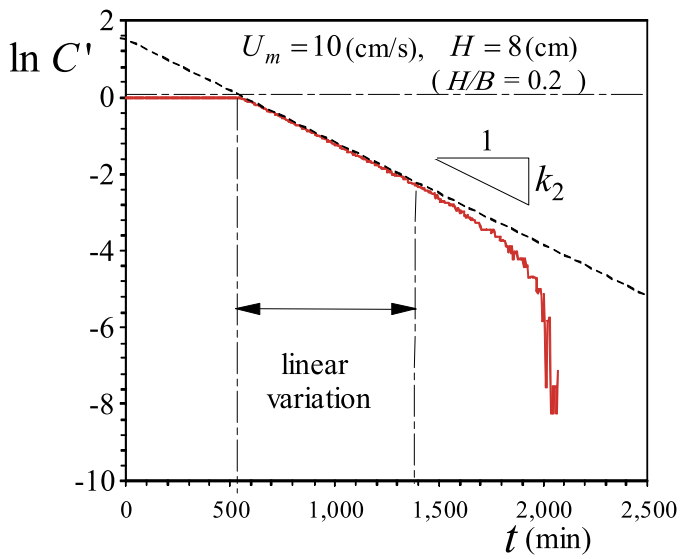

FIG. 11. Time-decay of normalized dissolved oxygen concentration. The reaeration coefficient $k_{2}$ is evaluated using the slope in the linear variation zone.

conducted a highly accurate turbulence measurement by using laser Doppler anemometer, and revealed there are universal functions of the vertical profiles for the turbulent intensities, turbulent kinetic energy, and eddy viscosity normalized by the friction velocity. Therefore, turbulence in an open-channel is found to be standardized by the bottom friction velocity, and this suggests that larger turbulence is transferred from the bottom toward the free surface for the higher-speed stream. It results in a variation in the gas transfer velocity corresponding to the bulk-mean velocity as seen in Fig. 12. In contrast, gas transfer is found to be more effective for the larger depth condition. Figure 13 shows the variation of the gas transfer velocity normalized by the friction velocity, $k_{L}{ }^{+}$, with the normalized depth, $H / B . k_{L}{ }^{+}$is found to depend more strongly on the water depth rather than the bulk-mean velocity. In particular, there is a strong linear relationship between $k_{L}{ }^{+}$and $H / B$. This is because friction velocity is not driven by secondary currents as compared with the gas transfer velocity. The variation of $U_{*}$ is comparatively small, irrespective of the water depth. This implies that not only friction velocity but also details of the

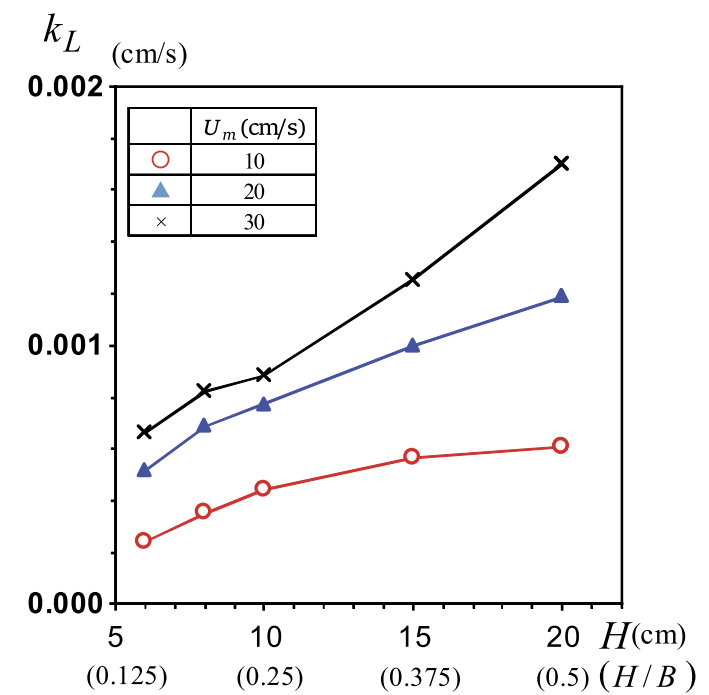

FIG. 12. Relationship between gas transfer velocity and water depth. The interfacial gas transfer is enhanced corresponding to $U_{m}$ under the same depth condition.

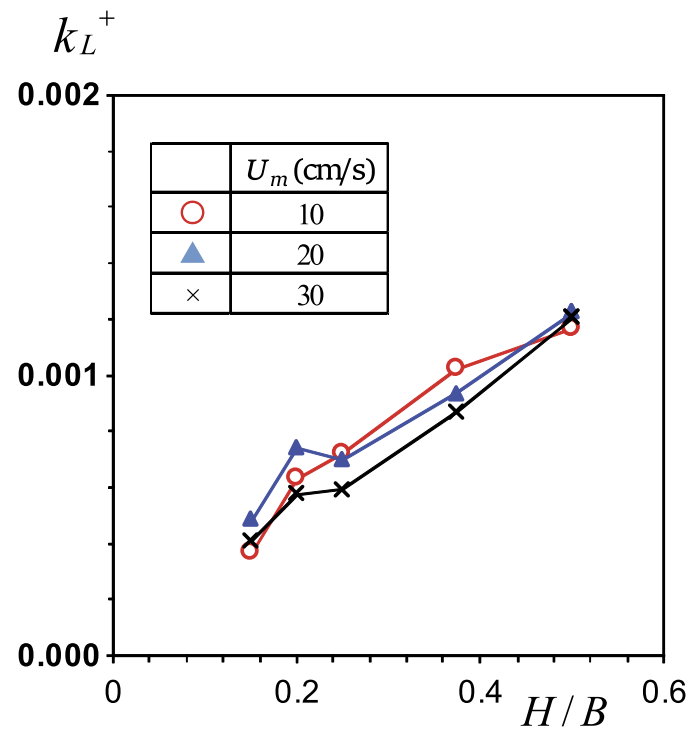

FIG. 13. Relationship between normalized gas transfer velocity and normalized depth. There is a strong linear variation and $k_{L}{ }^{+}$depends more significantly on the depth than on the bulk-mean velocity. This result suggests that there is the possibility that the gas transfer is controlled by secondary currents.

water depth and aspect ratio are needed to evaluate accurately gas transfer velocities.

Secondary currents are formed due to there being walls on three sides of the flow cross section; as these are able to fill a greater percentage of the volume at greater depths, both the gas transfer and vertical mass transport will be enhanced. The variation of the water depth is exactly equivalent to that of the aspect ratio under the fixed flume-width condition. Lateral currents develop notably with large-scale cross-sectional circulation for large depth, i.e., small aspect ratio instances as indicated in Fig. 3. Not only bottom-situated turbulent bursting but also secondary currents are expected to make interfacial gas transfer more effective.

Indeed, many conventional formulae are power functions of the characteristic velocity such as $U_{*}$ and $U_{m}$, water depth, and bed slope. The coefficients are quite different among the suggested formulae because reliable measurements of friction velocity and bed slope are very difficult to attain for natural rivers. In contrast, friction velocity is not required in the SD model. Even if digital images with corresponding velocity vectors on the free surface were prepared, the SD model readily gives $k_{L}$. This is very attractive and motivated the present study.

\section{Examination of the SD model}

\section{Original SD model}

Equation (3) proposed by McCready et al. ${ }^{2}$ is rewritten using a coefficient of proportionality $\alpha$,

$$
k_{L}=\alpha \sqrt{D \beta^{\prime}} .
$$

Turney and Banerjee ${ }^{5}$ reviewed the coefficient of proportionality $\alpha$ which previous researchers evaluated for various kinds of flow fields, i.e., oscillation tank, open-channel flow, and wind-driven current. Although their results obey the linear relation Eq. (24), $\alpha$ is not constant but distributes over a wide range $(0.2-0.7)$. This fact suggests that this variation is caused 
not only by the kind of flow field but also the experimental environment.

Figure 14 compares the relationship between $k_{L}$ and $\beta^{\prime}$ measured in a grid-stirred tank and in wind-induced water waves. At fixed water depth, the relationship is linear for both the present and previous studies. The present data are located within the range of $\alpha(0.1-0.25)$ which corresponds with a previous result for an open channel measured by Turney and Banerjee $^{5}(\alpha=0.25)$. Therefore, the present coefficient of proportionality corresponds to those determined in other kinds of flow fields. In contrast, the slope of the linear relation depends on the water depth, in which a coefficient of determination $R^{2}$ is 0.45 . Hence, the SD model must be modified to characterize open-channel flows such as natural rivers. A comparison of Eqs. (12) and (24) suggests that $\alpha$ includes the normalized parameter accounting for large and small scales, and it induces a notable dependence on the water depth. The variability of $\alpha$ seen in previous studies may also be related to the influence of $L^{+}$.

\section{Modified SD model}

In modifying the SD model, we define a normalized length $L^{+}$introduced in Eq. (12). Recall that $L^{+}$includes a characteristic velocity and length for the water-depth-scale vortex which are related to interfacial gas transfer. The secondary currents promote convection diffusion and turbulence transport. The increased SR rate, driven by these currents and the large-scale coherent vortices, is quantified by the $L^{+}$index. As mentioned above, the coefficient of proportionality $\alpha$ in the original SD model depends on the water depth. In the proposed model given by Eq. (12), the water depth is the characteristic length scale.

Figure 15 gives the gas transfer velocity as a function of $\sqrt{L^{+} D \beta^{\prime}}$. For all tested water depths, the relationship is linear and is clearly recognizable. The coefficient of determination $R^{2}$ is 0.68 , which is an improvement over that for the original

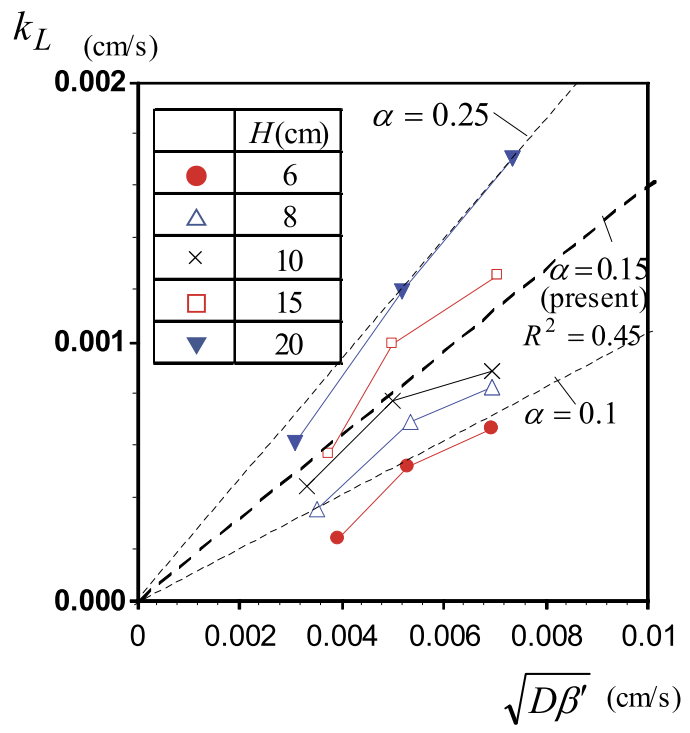

FIG. 14. Examination of original surface divergence model; comparison of proportionality coefficient in different depth cases. The slope of the linear relation depends on the water depth.

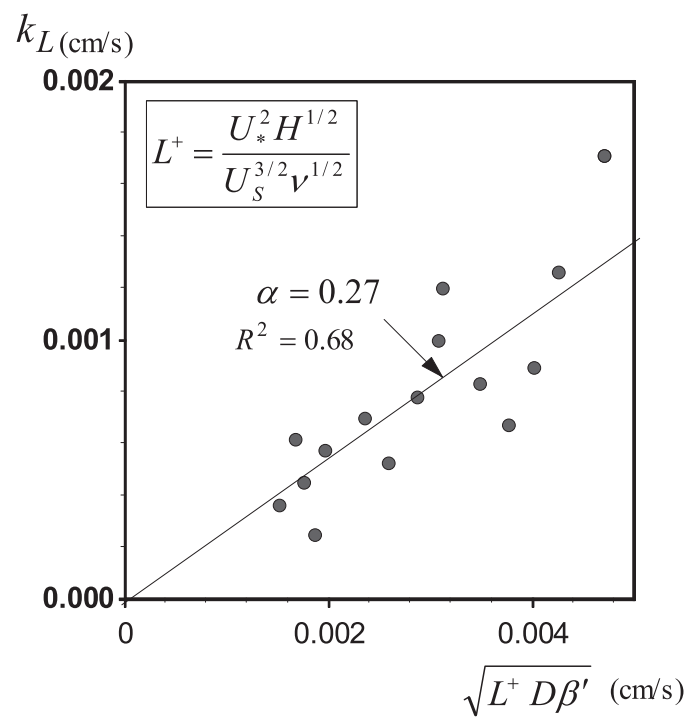

FIG. 15. Linear relationship given by modified SD models with using friction velocity. The coefficient of determination $R^{2}=0.68$ which is improvement over that for the original SD model $\left(R^{2}=0.45\right)$.

SD model (0.45). Consequently, for determining the gas transfer velocity in open-channel flows, we propose the empirical formula

$$
k_{L} / \sqrt{L^{+} D \beta^{\prime}}=0.27, \quad L^{+}=\left(\frac{U_{*}^{2} H^{1 / 2}}{v^{1 / 2} U_{s}^{3 / 2}}\right) .
$$

Figure 16 shows the relationship between the gas transfer velocity and $\sqrt{L^{+} D \beta^{\prime}}$, in which $U_{*}$ is subtracted by Eq. (22). The fitting function is obtained in a same manner to Eq. (25),

$$
k_{L} / \sqrt{L^{+} D \beta^{\prime}}=0.89, \quad L^{+}=\left(\frac{\beta^{\prime} v^{3 / 10} H^{7 / 10}}{U_{s}^{13 / 10}}\right) .
$$

The coefficient of determination is 0.82 as indicated in the figure; this is much better than that of the original SD model.

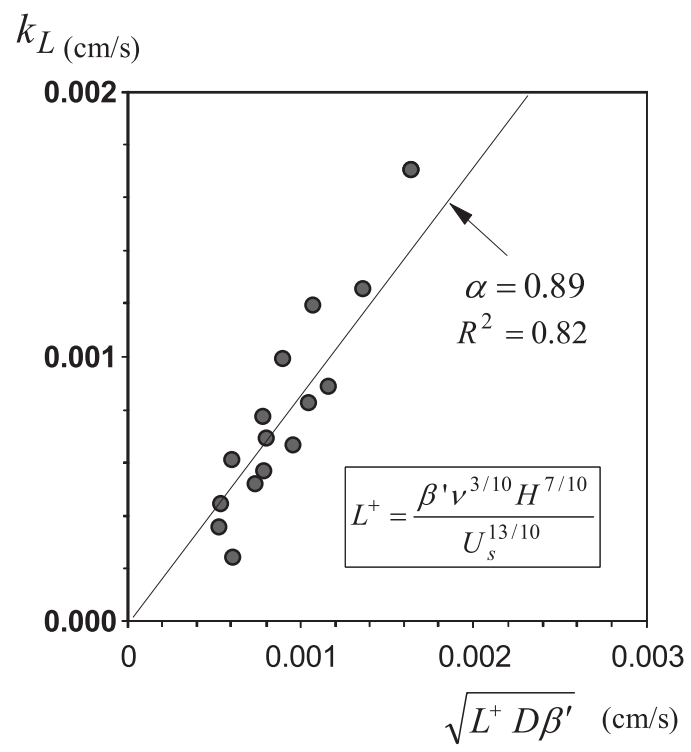

FIG. 16. Linear relationship given by modified SD models without friction velocity. Subtracting $U_{*}$ by the fitting curve in Fig. $10, R^{2}$ goes up to 0.82 . The modified SD model of Eq. (26) proved effective without the dependence on bulk mean velocity and water depth, and has a larger coefficient of determination than the original SD model. 
Together with the bottom bursting events, the large-scale coherent vortices are expected to promote interfacial gas transfer and also depth-scale secondary currents possessing a longitudinal axis. They also increase the SR rate by inducing significant convection diffusion and turbulent mixing of dissolved oxygen. The slopes in Figs. 15 and 16 depend much less on the water depth in the modified model involving $L^{+}$ than in the SD model of McCready et al. ${ }^{2}$ A following form is introduced to consider contribution of depth-scale currents to the interfacial gas transfer:

$$
\begin{aligned}
L^{+} & =\frac{U_{*}^{2} L_{H}^{1 / 2}}{v^{1 / 2} U_{H}^{3 / 2}}=v^{1 / 2}\left(U_{*} \frac{U_{*}}{v}\right) \times \frac{1}{L_{H}^{1 / 2} U_{H}^{1 / 2}} \times \frac{L_{H}}{U_{H}} \\
& =\left(\frac{v}{U_{H} L_{H}}\right)^{1 / 2} \times\left(U_{c} \frac{U_{c}}{v}\right) \times \frac{L_{H}}{U_{H}} \\
& =\left(\frac{v}{U_{s} H}\right)^{1 / 2} \times \frac{U_{c}}{L_{c}} \times \frac{L_{H}}{U_{H}}=\frac{1}{\operatorname{Re}_{s}^{1 / 2}} \times \frac{T_{H}}{T_{c}},
\end{aligned}
$$

in which $T_{H} \equiv L_{H} / U_{H}$ is a characteristic time of large-scale events. Eq. (27) means that $L^{+}$is a ratio of a dynamic range of large and small turbulence events $T_{H} / T_{c}$ to the Reynolds number $\mathrm{Re}_{s}$, that is, a parameter related to the contributions of depth-scale large vortices to interfacial gas transfer in fully developed turbulent open-channel flows defined by a certain Reynolds number. Together with bottom bursting events, large-scale coherent vortices are expected to promote interfacial gas transfer and also the depth-scale secondary currents. They also increase the SR rate by inducing significant convection diffusion and turbulent mixing of dissolved oxygen.

Thackston and $\mathrm{Krenkel}^{8}$ developed a very useful model, in which $k_{2}$ is proportional to $U_{*} / H$. The well-known correspondence between $U_{*}$ and bed turbulence can be inferred from the turbulence statistics normalized by $U_{*}$, which obey the universal function proposed by Nezu and Nakagawa ${ }^{7}$ in the equilibrium layer. $U_{*}$ is closely related to the freesurface turbulence factors $\beta^{\prime}$ and $t_{k}$ through diffusion in the mid-depth region. However, unlike the model of Thackson and Krenkel, our model directly predicts gas transfer rates from free-surface turbulence. $U_{*}$ is difficult to measure accurately in non-uniform rivers. In contrast, for natural rivers, local values of $\beta^{\prime}$ used in Eq. (26) are easily obtained using current image sensing techniques such as PIV.

\section{CONCLUSIONS}

We have focused on the air/water gas transfer phenomenon in open-channel flows. The applicability of the surface velocity divergence (SD) model was examined, and the SD model was found to depend on the water depth. Our new model is applicable for different water depth cases, i.e., there is a linear relationship between the gas transfer velocity and surface velocity divergence in open-channel flows. The main results of this study are summarized below.

Laboratory measurements were conducted systematically varying the bulk-mean velocity and the water depth to reveal the relationship between the gas transfer velocity and surface velocity divergence. The measurement results revealed that the coefficient of proportionality of the original SD model depends significantly on the water depth.

HPIV measurements provide details of the instantaneous divergence of the free-surface velocity components. The positive and negative regions of divergence were transferred downstream with time, as occurs in the boil phenomenon on natural river surfaces. This implies that interfacial gas transfer is related to bottom-situated turbulence motion and vertical mass transfer.

The gas transfer velocity normalized by the friction velocity was found to depend more significantly on the water depth rather than the bulk-mean velocity. In particular, there is a strong linear relationship between normalized the gas transfer velocity and aspect ratio. This is because the friction velocity is not driven by secondary currents compared with the gas transfer velocity.

The original SD model focuses mainly on viscous smallscale vortex motion, and there is a possibility that the depth dependence of the SD model results from this fact. Therefore, a non-dimensional parameter incorporating the characteristic velocity and length scales on both small-scale and large-scale motions is introduced into the analysis. The SD model was modified using this parameter to accommodate the effects of the water depth on gas transfer. The modified SD model proved effective without the dependence on the bulk mean velocity and the water depth, and has a larger coefficient of determination than the original SD model.

The proposed SD model is expected to evaluate gas transfer velocities in small rivers, where depth information is relatively easy to acquire. In contrast, in large rivers and oceans, the water depth must be replaced by an appropriate length scale. In the near future, we shall improve the present modified SD model to reasonably predict the gas transfer velocity in the various types of flow fields.

\section{ACKNOWLEDGMENTS}

The authors would like very much to thank the editors of Physics of Fluids and anonymous reviewers for very valuable and encouraging comments. The present experiments were carried out under the support from the graduate school students, Mr. Yuta Tanaka, Mr. Kazuya Takahashi, and Mr. Shinya Gotou. The authors gratefully acknowledge this support.

\section{NOMENCLATURE}

$\begin{array}{ll}\alpha & \text { proportionality coefficient } \\ \beta^{\prime+} & \begin{array}{l}\text { normalized surface velocity diver- } \\ \text { gence }\end{array} \\ \tilde{\beta} & \begin{array}{l}\text { instantaneous surface velocity diver- } \\ \text { gence }\end{array} \\ \beta^{\prime} & \begin{array}{l}\text { intensity of surface velocity diver- } \\ \text { gence evaluated by RMS operation }\end{array} \\ \varepsilon & \text { turbulent energy dissipation rate } \\ \tau_{b} & \text { bed shear stress } \\ \rho & \text { water density } \\ \mu & \text { viscosity }\end{array}$




$v$
$\Pi_{\beta^{\prime}} \equiv v \beta^{\prime} / U_{s}^{2}$,
$\Pi_{H} \equiv U_{s} H / v=\operatorname{Re}_{s}$
$k_{L}$
$k_{L}^{+}$
$k_{2}$
$t_{k} \equiv\left(u^{\prime 2}+w^{2}\right) / 2$
$x$
$x_{d}$
$y$
$z$
$y^{\prime}$
$y^{+} \equiv y U_{*} / v$
$\tilde{u}_{r}$
$u$
$v^{\prime}$
$w^{\prime}$
$u^{\prime}$

$$
\tilde{w}_{r}
$$

$B$

$C^{\prime} \equiv\left(C_{s}-C\right) /\left(C_{s}-C_{0}\right)$

C

$C_{S}$

$C_{0}$

$\tilde{c}$

$D$

$H$

$L^{+}$ kinematic viscosity.

non-dimensional parameter in pie theorem

non-dimensional parameter in pie

theorem

gas transfer velocity

normalized gas transfer velocity

reaeration coefficient

turbulent kinetic energy at free-

surface

streamwise coordinate

developing distance of boundary

layer from channel entrance

vertical coordinate

spanwise coordinate

distance from the free-surface in

the direction of the flume bottom

vertical coordinate normalized by inner variables

turbulence component in streamwise direction

turbulence component in vertical direction

turbulence component in spanwise direction

turbulence intensity in streamwise direction

turbulence intensity in vertical direction

turbulence intensity in spanwise direction

instantaneous velocity component in streamwise direction

instantaneous velocity component in vertical direction

instantaneous velocity component in spanwise direction

instantaneous streamwise velocity in specific large divergence/ convergence location used for reference velocity scale

instantaneous spanwise velocity in specific large divergence/ convergence location used for reference velocity scale

channel width

normalized dissolved oxygen concentration

dissolved oxygen concentration

saturated dissolved oxygen concentration

initial dissolved oxygen concentration

instantaneous dissolved oxygen concentration

diffusion coefficient of dissolved oxygen

water depth

non-dimensional length factor
$\mathrm{Fr} \equiv U_{m} / \sqrt{g H} \quad$ Froude number

$R e \equiv U_{m} H / v \quad$ Reynolds number using bulk-mean velocity

$R e_{s} \equiv U_{s} H / v \quad$ Reynolds number using surface velocity

$S \equiv v / D$

$U$

V

$W$

$U_{c}$

$T_{c}$

$L_{c}$

$U_{H}$

$T_{H}$

$L_{H}$

$U_{*}$

$U_{m}$

$U_{s}$

Schmidt number

time-averaged velocity in streamwise direction

time-averaged velocity in vertical direction time-averaged velocity in spanwise direction small-scale characteristic velocity small-scale characteristic time small-scale characteristic length large-scale characteristic velocity large-scale characteristic time large-scale characteristic length bottom friction velocity bulk-mean velocity time-averaged streamwise velocity at freesurface

${ }^{1}$ P. V. Danckwerts, "Significance of liquid-film coefficients in gas absorption," Ind. Eng. Chem. 43, 1460-1467 (1951).

${ }^{2}$ M. J. McCready, E. Vassiliadou, and T. J. Hanratty, "Computer simulation of turbulent mass transfer at a mobile interface," AIChE J. 32, 1108-1115 (1986).

${ }^{3}$ A. Tamburrino and J. S. Gulliver, "Free-surface turbulence and mass transfer in a channel flow," AIChE J. 48(12), 2732-2743 (2002).

${ }^{4}$ S. Banerjee, D. Lakehal, and M. Fulgosi, "Surface divergence models for scalar exchange between turbulent streams," Int. J. Multiphase Flow 30, 963-977 (2004).

${ }^{5}$ D. E. Turney and S. Banerjee, "Air-water gas transfer and near-surface motions," J. Fluid Mech. 733, 588-624 (2013).

${ }^{6} \mathrm{~S}$. K. Robinson, "Coherent motions in the turbulent boundary layer," Annu. Rev. Fluid Mech. 23, 601-639 (1991).

${ }^{7}$ I. Nezu and H. Nakagawa, Turbulence in Open-Channel Flows, IAHR Monograph (Balkema, 1993).

${ }^{8}$ E. L. Thackston and P. A. Krenkel, "Reaeration prediction in natural streams,” J. Sanit. Eng. Div. 95, 65-94 (1969).

${ }^{9}$ J. S. Gulliver and M. J. Halverson, "Air-water gas transfer in open channels," Water Resour. Res. 25(8), 1783-1793, doi:10.1029/wr025i008p01783 (1989).

${ }^{10} \mathrm{~S}$. Komori, Y. Murakami, and H. Ueda, "The relationship between surfacerenewal and bursting motions in an open-channel flow," J. Fluid Mech. 203, 103-123 (1989).

${ }^{11}$ D. B. Moog and G. H. Jirka, "Air-water gas transfer in uniform channel flow," J. Hydraul. Eng. 125(1), 3-10 (1999).

${ }^{12}$ H. M. Nepf and E. R. Vivoni, "Flow structure in depth-limited, vegetated flow," J. Geophys. Res. 105, 28547-28557, doi:10.1029/2000jc900145 (2000).

${ }^{13}$ C. A. M. E. Wilson, T. Stoesser, P. D. Bates, and A. Batemann Pinzen, "Open channel flow through different forms of submerged flexible vegetation," J. Hydraul. Eng. 129, 847-853 (2003).

${ }^{14}$ D. Poggi, A. Porpotato, and L. Ridolfi, "The effect of vegetation density on canopy sub-layer turbulence," Boundary-Layer Meteorol. 111, 565-587 (2004)

${ }^{15}$ F. G. Carollo, V. Ferro, and D. Termini, "Flow resistance law in channel with flexible submerged vegetation," J. Hydraul. Eng. 131, 554-564 (2005).

${ }^{16} \mathrm{M}$. Ghisalberti and H. M. Nepf, "The structure of the shear layer in flows over rigid and flexible canopies," Environ. Fluid Mech. 6, 277-301 (2006).

${ }^{17}$ H. M. Nepf, M. Ghisalberti, B. White, and E. Murphy, "Retention time and dispersion associated with submerged aquatic canopies,"Water Resour. Res. 43, W04422, doi:10.1029/2006wr005362 (2007).

${ }^{18} \mathrm{I}$. Nezu and M. Sanjou, "Turbulence structure and coherent motion in vegetated open-channel flows," J. Hydro-Environ. Res. 2, 62-90 (2008).

${ }^{19}$ A. N. Sukhodolov and T. A. Sukhodolova, "Case study: Effect of submerged aquatic plants on turbulence structure in a lowland river," J. Hydraul. Eng. 136, 434-446 (2010).

${ }^{20} \mathrm{~T}$. Okamoto, I. Nezu, and H. Ikeda, "Vertical mass and momentum transport in open-channel flows with submerged vegetations," J. Hydro-Environ. Res. 6(4), 287-297 (2012). 
${ }^{21}$ D. E. Turney, W. C. Smith, and S. Banerjee, "A measure of nearsurface fluid motions that predicts air-water gas transfer in a wide range conditions," Geophys. Res. Lett. 32, L04607, doi:10.1029/2004gl021671 (2005).

${ }^{22}$ Y. Hasegawa and N. Kasagi, "Systematic analysis of high Schmidt number turbulent mass transfer across clean, contaminated and solid interfaces," Int. J. Heat Mass Transfer 29, 765-773 (2008).

${ }^{23}$ I. Calmet and J. Magnaudet, "Statistical structure of high Reynolds-number turbulence close to free surface of an open-channel flow," J. Fluid Mech. 474, 355-378 (2003).

${ }^{24}$ C. N. S. Law and B. C. Khoo, "Transport across turbulent air-water interface," AIChE J. 48, 1856-1868 (2002).

${ }^{25}$ S. P. McKenna and W. R. McGillis, "The role of free-surface turbulence and surfactants in air-water gas transfer," Int. J. Heat Mass Transfer 47, 539-553 (2004).

${ }^{26} \mathrm{Z}$. F. Xu and B. C. Khoo, "Mass transfer across the turbulent gas-water interface," AIChE J. 52, 3363-3374 (2006).
${ }^{27}$ H. Tsumori and Y. Sugihara, "Length scales of motions that control air/water gas transfer in grid stirred turbulence," J. Mar. Syst. 66, 6-18 (2007).

${ }^{28}$ Herlina and G. H. Jirka, "Experiments on gas transfer at the air-water interface induced by oscillating grid trubulence," J. Fluid Mech. 594, 183-208 (2008).

${ }^{29} \mathrm{H}$. Tennekes and J. L. Lumley, First Course in Turbulence (MIT Press, 1972).

${ }^{30}$ M. S. Kirlkgoz and M. Ardichoglu, "Velocity profiles of developing and developed open channel flow," J. Hydraul. Eng. 123(12), 1099-1105 (1997).

${ }^{31}$ I. Nezu and W. Rodi, "Experimental study on secondary currents in open channel flow," Proceedings of the 21st IAHR Congress, Melbourne (IAHR, 1985), Vol. 2, pp. 19-23.

${ }^{32} \mathrm{H}$. Khang and S. U. Choi, "Reynolds stress modelling of rectangular openchannel flow,” Int. J. Numer. Methods Fluids 51, 1319-1334 (2006).

${ }^{33}$ I. Nezu and W. Rodi, "Open-channel flow measurements with a laser Doppler anemometer," J. Hydraul. Eng. 112(5), 335-355 (1986). 